\title{
The microbial aetiology of genital ulcers in black men in Durban, South Africa
}

\author{
Y M COOVADIA, A KHARSANY, AND A HOOSEN \\ From the Department of Microbiology, Faculty of Medicine, University of Natal, Durban, South Africa
}

SUMMARY The microbial aetiology of genital ulcers was assessed in 100 black men attending a sexually transmitted disease (STD) clinic in Durban, South Africa. Forty patients harboured Haemophilus ducreyi, nine herpes simplex virus, and one Neisseria gonorrhoeae. Syphilis was diagnosed in 44 patients on the basis of dark field microscopy or positive syphilis serology test results, or both. Of these 44 patients, eight also harboured $H$ ducreyi, one herpes simplex virus. Lymphogranuloma venereum was diagnosed in one patient. No cause of ulceration could be found in the remaining 16 patients.

\section{Introduction}

Reliable statistics on the incidence of sexually transmitted diseases (STDs) in South Africa are not readily available. Nevertheless, the incidence is probably ten to twelve times that in the United Kingdom. The STD clinic at Congella, Durban sees an average of 25000 new cases yearly. Gonorrhoea and syphilis are two of the most common STDs treated at this clinic. Because of limited laboratory facilities, the microbial diagnosis of genital ulcers is based on clinical appearances alone. In addition the results of serological tests for syphilis are only available a few days later. Recent studies in Swaziland, ${ }^{1}$ Johannesburg, South Africa, ${ }^{2}$ and Nairobi, Kenya ${ }^{3}$ have indicated that Haemophilus ducreyi in the most common pathogen found in genital ulcers. Penicillins and tetracycline have also generally been reported as being ineffective in treating chancroid. ${ }^{23}$

In the report published here we discuss the microbial aetiology of genital ulcers in black men in Durban and also provide some guidelines for rational treatment with antimicrobials.

\section{Patients and methods}

\section{PATIENTS}

Black men with genital ulcers were examined at the STD clinic in Congella, Durban. We studied 100

Address for reprints: Dr Y M Coovadia, Department of Microbiology, University of Natal, PO Box 17039, Congella 4013, Durban, South Africa

Accepted for publication 13 December 1984 patients who attended the clinic between 18 and 26 June 1984 who had not received antibiotic treatment in the previous four weeks. A detailed history was obtained, and the genitalia and regional lymph nodes were examined carefully.

\section{LABORATORY INVESTIGATIONS}

Exudate from the base of ulcers was examined by dark field microscopy for Treponema pallidum, and smears were prepared on sterile glass slides for Gram staining for $H$ ducreyi and Giemsa staining for Calymmatobacterium granulomatis. Two sterile calcium alginate tipped swabs were used to obtain material from the bases and margins of ulcers. The first swab was inoculated on to Müeller-Hinton (Baltimore Biological Laboratories (BBL, Cockeysville, Maryland, USA)) agar plates (supplemented with $5 \%$ sterile horse blood heated to $75^{\circ} \mathrm{C}, 1 \%$ IsoVitaleX (BBL), and $3 \mathrm{mg} / \mathrm{l}$ vancomycin) to isolate $\boldsymbol{H}$ ducreyi. These plates were immediately placed in candle jars and transported to the laboratory within two hours, where they were transferred into Gas Pak jars (BBL) without a catalyst and incubated under microaerophilic conditions at $37^{\circ} \mathrm{C}$. The plates were examined at 48 hours and thereafter daily for two weeks. $H$ ducreyi was identified by the morphological and biochemical criteria described by Killian ${ }^{4}$ and Hammond et al. ${ }^{5}$ The isolates were screened for $\beta$ lactamase production by the chromogenic cephalosporin test ${ }^{6}$ and for the presence of cytochrome oxidase by the addition of a few drops of $1 \%$ tetramethyl-p-phenylenediamine dihydrochloride (BDH Chemicals, Poole, Dorset, England) direct to the colonies. The second swab was immediately placed in viral transport medium consisting of Eagle's 
minimum essential medium containing $10 \%$ fetal calf serum, $1000 \mathrm{mg} / \mathrm{l}$ penicillin, and $100 \mathrm{mg} / \mathrm{l}$ streptomycin, and kept on ice until transferred to the laboratory. The specimens were then inoculated on to rabbit kidney cells (RK cells, MA Bioproducts, Maryland, USA), which were incubated at $37^{\circ} \mathrm{C}$ and observed daily for two weeks. Herpes simplex virus was identified by its characteristic cytopathic effects, and its identity was confirmed by staining with fluorescein isothiocyanate labelled antisera against herpes simplex virus types 1 and 2 (MA Bioproducts, Maryland, USA). Intraurethral secretions were obtained from all patients using calcium alginate tipped swabs, which were then inoculated on to modified New York City medium (Oxoid, Basingstoke, Hampshire, England) and incubated in candle extinction jars at $37^{\circ} \mathrm{C}$. Serum samples were obtained from all patients and tested by the rapid plasma reagin (RPR) and by the fluorescent treponemal antibody absorbed (FTA-ABS) tests. Antibodies to herpes simplex virus types 1 and 2 and to chlamydiae were detected by commercially available enzyme linked immunosorbent assay (ELISA) kits (MA Bioproducts). These tests were performed in accordance with instructions supplied with each kit and included positive and negative controls. A single serum specimen from each patient was tested.

In this study no attempt was made to isolate chlamydiae from either the genital ulcers or buboes. The diagnosis of lymphogranuloma venereum was based on clinical grounds alone.

\section{Results}

\section{CLINICAL FINDINGS}

The 100 patients studied were aged 17 to 51 (mean $26 \cdot 6$ ) years, 80 of whom were aged 16 to 32 . Only five patients were circumcised. In most cases the source of infection was casual sexual contacts. A history of genital ulcers was obtained from 31 patients, and 35 had had more than one sexual contact in the previous month. The incubation period of ulcers varied from one day to more than a month. The most common sites for penile ulceration were the coronal sulcus and prepuce. Fifty patients had multiple ulcers, and 48 complained of painful ulcers. Inguinal lymphadenopathy was present in 55 patients and was bilateral in 18 of them. Sixteen patients had fluctuant buboes.

\section{LABORATORY FINDINGS}

The table shows that $H$ ducreyi was isolated from genital ulcers in 40 patients, two of whom also harboured herpes simplex virus and eight also had syphilis. Direct Gram staining of material from the ulcers did not help in identifying patients with
TABLE Microbial aetiology of genital ulcers in 100 black men

No of men whose ulcers contained:

\begin{tabular}{lr}
\hline Single infections: & \\
Haemophilus ducreyi only & 30 \\
Herpes simplex virus (HSV) only & 6 \\
Neisseria gonorrhoeae only & 1 \\
Treponema pallidum only & 35 \\
Lymphogranuloma venereum only & 1 \\
Mixed infections: & \\
H ducreyi and HSV & 2 \\
H ducreyi and T pallidum & 8 \\
HSV and T pallidum & 1 \\
No pathogens & 16 \\
\hline
\end{tabular}

chancroid. Only $11(28 \%)$ of the 40 patients with chancroid had characteristic Gram negative bacilli on direct smears from ulcers. All the isolates produced $\beta$ lactamase, and 97 contained cytochrome oxidase, although none contained cytochrome oxidase when tested by the Pathotec Cytochrome Oxidase Strips (General Diagnostics, New Jersey, USA). Of the $\mathbf{4 0}$ strains of $\boldsymbol{H}$ ducreyi tested by the Stokes agar disc diffusion method, $35(88 \%)$ were resistant to sulphonamide and $25(63 \%)$ were resistant to tetracycline. All strains tested were sensitive to erythromycin and sulphamethoxazole-trimethoprim.

Herpes simplex virus was detected in nine patients, two of whom were also infected with $H$ ducreyi and one was positive for $T$ pallidum on dark field microscopy. In the remaining six patients herpes simplex virus was the only pathogen detected.

Forty four patients had $T$ pallidum on dark field microscopy or positive results to RPR and FTA-ABS tests, or both. Dark field microscopy alone was positive in nine, and in 14 other patients it was positive together with RPR and FTA-ABS tests. In the remaining 21 patients, the diagnosis of syphilis was based on reactive RPR and FTA-ABS tests. Eight patients with syphilis had simultaneous infections with $H$ ducreyi and another with herpes simplex virus. Syphilis was the only diagnosis in the remaining 35 patients.

$N$ gonorrhoeae was isolated from the urethral swabs of nine patients. It was also isolated from the ulcer of one patient in whom simultaneous urethral cultures were negative. Gonococcal urethritis was associated with chancroid in five patients, syphilis in three, and herpes simplex in one.

Microscopical examination did not show typical Donovan bodies in any Giemsa stained smears. Lymphogranuloma venereum was diagnosed clinically in one patient who presented with multiple sinuses and massive enlargement of inguinal lymph nodes. The ELISA showed that this patient's serum was strongly positive for chlamydial antibodies, 
which were detected in 89 patients. The ELISA for herpes showed that 97 patients had detectable antibodies to herpes simplex type 2 , and all had antibodies to herpes simplex type 1 . A positive result to this test is equivalent to an indirect haemagglutination titre of $\geqslant 1 / 8$ or to a virus neutralisation titre of $\geqslant 1 / 20$.

\section{Discussion}

Sexually acquired genital ulceration is common in black patients attending STD clinics in this country. ${ }^{2}$ Because of the lack of laboratory facilities at most of these clinics, the microbial diagnosis of genital ulcers is usually based on clinical criteria alone. Our findings indicate that clinical and epidemiological features alone are not reliable in diagnosing the aetiology of genital ulcers. Multiple painful ulcers, which are said to be characteristic of chancroid, were also noted in $36 \%(16 / 44)$ of patients with syphilis and in only $60 \%(24 / 40)$ of those with chancroid. The presence of buboes and the length of incubation period proved to be of some help in diagnosing chancroid. Of the 16 patients with buboes, $14(88 \%)$ were infected with $H$ ducreyi, and the mean incubation period for chancroid was seven days compared with 15 days for syphilis. Many of the patients proved ignorant of the fact that their ulcers had been sexually acquired. They attributed the ulcers to other causes, including "bewitchment".

The study published here shows that syphilis and chancroid together accounted for more than $80 \%$ of genital ulcers in our study population. Syphilis was present in $44 \%$ of patients, which is similar to the 49\% reported in Pretoria, South Africa, ${ }^{7}$ but is much higher than the $11 \%$ in Kenya, ${ }^{3} 17 \%$ in Denver, United States, ${ }^{8}$ and $0.6 \%$ in Sheffield, England. ${ }^{9}$ Dark field microscopy detected $52 \%(23 / 44)$ of all cases of syphilis, the remainder having been diagnosed by positive reactions to RPR and FTAABS tests. These findings confirm that dark field microscopy in the hands of an experienced technologist, provides a simple, inexpensive, and rapid diagnosis of syphilis.

$H$ ducreyi was isolated in $40 \%$ of our patients compared with $62 \%$ in Kenya, ${ }^{3} 79 \%$ in Sheffield, England, ${ }^{9}$ and $45 \%$ in Pretoria, $^{7}$ and $61 \%$ in Johannesburg. ${ }^{2}$ As other workers have reported, ${ }^{3}$ we found that microscopical examination of Gram stained smears did not help identify patients with chancroid. In addition, we also found that different brands of Müeller-Hinton media varied tremendously in their ability to support the growth of $H$ ducreyi, with that supplied by BBL giving the best results. In contrast to strains of $\boldsymbol{H}$ ducreyi isolated in England, ${ }^{9}$ all our isolates were $\beta$ lactamase producing. Most of the isolates were also resistant to sulphonamides and tetracycline. All strains were fully susceptible to erythromycin and sulphamethoxazole-trimethoprim.

Herpes simplex virus was detected in only $9 \%$ of our patients, which compares with isolation rates of 4\% in $\mathrm{Kenya}^{3}$ and $9 \%$ in Johannesburg, South Africa, ${ }^{2}$ and contrasts with British ${ }^{9}$ and North American studies ${ }^{8}$ in which herpes simplex was the most common aetiological agent found in genital ulcers. Serological testing by ELISA showed that $97 \%$ of patients had detectable antibodies to herpes simplex virus type 2 . Similarly $89 \%$ had detectable antibodies to chlamydiae. It is therefore apparent that serological testing of single serum specimens for herpes and chlamydial antibodies is of little diagnostic value in this population.

More than one pathogen was detected in 11 patients, eight of whom had mixed infections with chancroid and syphilis, two chancroid and herpes, and one syphilis and herpes. Coexisting infections with $H$ ducreyi and syphilis ${ }^{2}$ and also $H$ ducrey $i$ and herpes simplex virus ${ }^{9}$ have been documented previously.

By examining Giemsa stained smears of exudate from the base of ulcers, we were not able to detect any cases of granuloma inguinale. As we did not use the recommended method of examining squashed preparations of tissue from the ulcers, we could have missed some cases of infection with Calymmatobacterium granulomatis. Nevertheless our findings confirm those reported by other workers from Kenya and South Africa that granuloma inguinale is a rare cause of genital ulcers in black men. ${ }^{237}$ In this study we have shown that syphilis and chancroid are two of the most common causes of genital ulcers in black men in Durban, South Africa. We would therefore recommend that, in the absence of laboratory facilities, genital ulcers be treated with antimicrobial agents that will be effective against both these organisms. This can be easily achieved by combined treatment with benzathine penicillin in a single dose of $2.4 \mathrm{MU}$ and erythromycin or co-trimoxazole orally for 14 days.

We thank Fauzia Naidoo and C Pillay for their expert technical help, and staff members of the Special Clinic, Congella, Durban, for their cooperation and help. We also thank Dr M Richter, Medical Officer of Health, Durban, for granting us permission to undertake the study and allowing us to publish our findings.

\section{References}

1. Meheus A, Van Dyck E, Friedman F. Importance of genital infections in Swaziland. In: Proceedings of the First African Regional Conference on Sexually Transmitted Disease, Nigeria, 1979. Yaba, Nigeria: Nigerian Institute for Medical Research,
1981. 
2. Duncan MO, Bilgeri YR, Fehler HG, Ballard RC. The diagnosis of sexually acquired genital ulcerations in black patients in Johannesburg. Southern African Journal of Sexually Transmitted Diseases $1981 ; 1: 20-3$.

3. Nsanze H, Fast MV, D'Costa LJ, Tukei P, Curran J, Ronald A. Genital ulcers in Kenya: clinical and laboratory study. British Journal of Venereal Diseases 1981;57:378-81.

4. Killian M. A taxonomic study of the genus Haemophilus, with the proposal of a new species. J Gen Microbiol 1976;93:9-62.

5. Hammond GW, Lian CJ, Wilt JC, Ronald AR. Comparison of specimen collection and laboratory techniques for isolation of Haemophilus ducreyi. J Clin Microbiol 1978; 7:39-43.

6. O'Callaghan $\mathrm{CH}$, Morris A, Kirby SM, Shingler AH. Novel method for detection of $\beta$-lactamase by using a chromogenic cephalosporin substrate. Antimicrob Agents Chemother 1972; 1:283-8.
7. Crewe-Brown HH, Krige FK, Davel GH, et al. Genital ulceration in males at Ga-Rankuwa Hospital, Pretoria. S Afr Med J 1982;62:861-3.

8. Chapel T, Brown WJ, Jeffries C, Stewart JA. The microbiological flora of penile ulcerations. J Infect Dis 1978; 137:50-6.

9. Kinghorn GR, Hafiz S, McEntegart MG. Pathogenic microbial flora of genital ulcers in Sheffield with particular reference to herpes simplex virus and Haemophilus ducreyi. British Journal of Venereal Diseases 1982;58:377-80. 